\title{
Reconstruction of electron beam energy spectra for vacuum and gas diodes
}

\author{
A.V. KOZYREV, V.YU. KOZHEVNIKOV, M.S. VOROBYOV, E.KH. BAKSHT, A.G. BURACHENKO, \\ N.N. KOVAL, AND V.F. TARASENKO \\ Institute High Current Electronics SB RAS, Tomsk, Russia \\ (RECEIVED 9 December 2014; ACCEPTED 20 February 2015)
}

\begin{abstract}
In this paper, the spectra of electron beams produced in vacuum and gas diodes were analyzed to study the capabilities and limitations of their reconstruction from beam attenuation in foils of different thickness. The electron energy distributions were calculated using the Tikhonov regularization for Fredholm integral equations on minimum a priori assumptions. The spectra reconstructed in the study were those of electron beams, including a supershort avalanche electron beam, produced in experiments on a DUET plasma-cathode electron accelerator and SLEP-150M accelerator.
\end{abstract}

Keywords: Electron beam attenuation in foils; Electron beam energy spectra; Spectrum reconstruction

\section{INTRODUCTION}

Electron beams with energies from tens to hundreds of kiloelectron-volts find wide application in various fields of science and technology (Mesyats, 2005; Li et al., 2006; Verbeeck et al., 2010). One of the most important parameters of electron beams is their energy spectrum. So the energy deposition to a gas in wide-aperture lasers with a vacuum diode was calculated with regard to electron beam absorption in its anode foil and scattering in the gas (Mesyats et al., 1995; Hegeler et al., 2002). However, similar calculations are timeconsuming and are normally applied to monoenergetic electron beams.

The generation of very short electron beams in vacuum diodes is such that a considerable portion of electrons is accelerated during the voltage rise and decay, and hence the spectra of nanosecond and subnanosecond electron beams are not monoenergetic. Moreover, electrons of energy $>$ $e U_{\mathrm{m}}$, where $e$ is the electron charge and $U_{\mathrm{m}}$ is the maximum gap voltage, are detected downstream of the anode foil of vacuum diodes at subnanosecond and nanosecond voltage pulse rise times (Khudyakova et al., 1964). The spectra of electron beams are measured with magnetic spectrometers (Bugaev et al., 1974; Mangles et al., 2004; Li et al., 2006) and time-of-flight spectrometers (Gloeckler \& Hsieh,

Address correspondence and reprint requests to: V.F. Tarasenko, Institute High Current Electronics SB RAS, Tomsk, Russia. E-mail: VFT@loi.hcei. tsc.ru
1979). The spectra of nano- and subnanosecond electron beams are also measured with the use of different methods (Bugaev et al., 1974; Afanasiev et al., 2005; Baksht et al., 2007), including the measurements of beam current attenuation in foils of different thickness (Baksht et al., 2010).

Now, the most divergent spectral measurement data are those for supershort avalanche electron beams (SAEBs) produced in point-to-plane gaps in atmospheric pressure air. According to our studies, the spectrum of a SAEB generated in atmospheric pressure air can sometimes reveal two to three groups of electrons differing in energy (Bakhst et al., 2008a; 2009; 2010; Tarasenko, 2011; Tarasenko et al., $2005 ; 2008 ; 2011 ; 2013)$. Electrons of the first (low-energy) group have kinetic energies $T_{1}<e U_{\mathrm{m}} / 3$; those of the second (main) group have energies $e U_{\mathrm{m}} / 3<T_{2}<e U_{\mathrm{m}}$; and electrons of the third group, which are termed electrons with "anomalous" energy (Babich \& Loiko, 1985), have energies $T_{3}>e U_{\mathrm{m}}$. The number of electrons in each group depends on experimental conditions, voltage pulse parameters, and cathode and gas diode designs. Increasing the number of electrons in the third group requires a decrease in voltage pulse rise time and an increase in cathode curvature radius. However, even under optimum SAEB generation conditions, the number of electrons of the third group is small and is not $>10 \%$ of the total number of electrons downstream of an $\mathrm{Al}$ anode foil $10 \mu \mathrm{m}$ thick (Baksht et al., 2010).

In earlier papers by Babich and Loiko (Babich \& Loiko, $1985 ; 1991)$, it is stated that with an interelectrode gap of 
$2 \mathrm{~cm}$ and gap voltage $U_{\mathrm{m}}$ equal to $190 \mathrm{kV}$, the maximum of the electron energy distribution in atmospheric pressure air corresponds to $290 \mathrm{keV}$ and most of the beam electrons have an energy $100 \mathrm{keV}$ higher than $e U_{\mathrm{m}}$. The same data are reported in their recent paper (Babich et al., 2014) in which, without any new research results, we have again presented the spectrum of runaway electrons obtained previously (Babich \& Loiko, 1985). Moreover, the statement that the runaway electron beam formed in atmospheric pressure air is almost monoenergetic (Babich et al., 2014) and is erroneous in our opinion, because the generation of a monoenergetic runaway electron beam varying at gap voltage and varying current through the gap is rather problematic. The data reported in the cited papers contradict both our results (Baksht et al., 2008a; 2008b; 2009; 2010; Tarasenko et al., $2005 ; 2008 ; 2011 ; 2013)$ and those reported elsewhere (Mesyats et al., 2011; Yatom et al., 2011). In particular, Mesyats et al. (2011) consider that the maximum energy for runaway electrons produced in atmospheric pressure air cannot exceed $e U_{\mathrm{m}}$ under any conditions. The foregoing suggests the necessity to improve the methods of spectral measurement for further research in runaway electrons and for diagnostics of ordinary electron beams.

Electron spectra can be determined by comparatively simple methods which give energies of the main groups of fast electrons. So, for example, electron spectra can be determined from electron absorption in foils of different thickness with the use of different variants of electron spectrum reconstruction. The simplest way is that, in which extrapolated ranges of electrons are found for characteristic portions of their absorption curve, and next the data on extrapolated ranges are summed up and the electron energy distribution is determined (Tarasenko et al., 2005; Baksht et al., 2008a).

A more complex method is that in which the initial electron beam is represented as a superposition of the $N$ number of monoenergetic electron groups with energy $E_{1}$, $E_{2}, E_{3}, \ldots, E_{N}$ and intensity $I_{1}, \ldots, I_{N}$ (Baksht et al., 2009). The intensity can be represented by the electron charge or electron beam current. As the electron beam passes through a barrier, its intensity decreases. So, to determine the number of electrons in each group, we have to select intensities of monoenergetic electrons at which their calculated and experimental attenuations in filters would be as close as possible. The selection allows one to find an electron spectrum such that the calculated and experimental electron beam attenuations per filter differ by $< \pm 15 \%$. However, the practical use shows that the electron spectrum reconstruction, in this case, admits the existence of several markedly different solutions.

Another method of spectral reconstruction of a short electron beam from its attenuation in foils of different thickness is proposed elsewhere (Baksht et al., 2010). The reconstruction is based on solving an ill-posed inverse problem by the Tikhonov regularization method which, on minimum a priory assumptions, gives unambiguous and physically substantiated results. The method was used for spectral reconstruction of runaway electrons generated in atmospheric pressure gases (Shao et al., 2012; Zhang et al., 2014). Of interest is to employ this method for spectral reconstruction of electron beams produced in vacuum diodes and to analyze its applicability to monoenergetic electron beams.

The main objectives of our study are the following: (1) analyzing the capabilities and limitations of reconstruction of electron beam spectra by the Tikhonov regularization method developed for solving ill-posed inverse problems (Fredholm integral equation of the first kind); (2) measuring the attenuation of microsecond and nanosecond electron beams produced in vacuum diodes and reconstructing their spectra from this attenuation by the regularization method; and (3) measuring the attenuation of subnanosecond electron beams and reconstructing their spectra from this attenuation by the same method.

\section{RECONSTRUCTION OF ELECTRON BEAM SPECTRA FROM BEAM ATTENUATION IN MATTER}

In the linear problem statement for a non-monoenergetic electron beam described by the distribution function $f(\epsilon)$ normalized to unity, the attenuation factor $g(x)$ should be calculated by the formula:

$$
g(x)=\int_{E_{\min }}^{E_{\max }} \tau(x, \varepsilon) f(\varepsilon) d \varepsilon,
$$

where $E_{\min }$ and $E_{\max }$ are the minimum and maximum electron energies in the spectrum of an incident beam; $\tau(\epsilon, x)$ is a known function of the attenuation factor for a monoenergetic electron beam with energy $\epsilon$ after its normal incidence on a plane layer of known material of thickness $x$.

First, we have to experimentally measure the dependence $g_{\text {exp }}(x)$ by the thin foil method and then to determine the function $f(\epsilon)$ from the data obtained. In other words, we have to find the solution $f(\epsilon)$ of integral Eq. (1) with known $\tau(\epsilon, x)$ from experimental data on $g_{\text {exp }}(x)$. However, experimental measurement of $g_{\text {exp }}(x)$ inevitably gives an error and formal methods developed for equations with exact initial data fail to provide the desired solution of the problem. The point is that the solution derived in this way for Fredholm integral Eq. (1) is unstable because even a relatively small change in the left-hand side of (1) greatly affects the exact solution $f(\epsilon)$. Therefore, similar mathematical problems are classified as ill-posed problems (Tikhonov \& Arsenin, 1977).

Although searching for a formally exact solution of (1) with an approximate left-hand side is meaningless, we can find its stable approximate solution on certain assumptions of the desired function $f(\epsilon)$. Let us briefly dwell on one of the methods for approximate solution of Eq. (1), namely on the Tikhonov regularization. 
In the Fredholm integral equation of the first-kind

$$
g_{\exp }(x)=\int_{a}^{b} K(x, s) f(s) d s \equiv K[f],
$$

the kernel $K(x, s)$ be uniquely defined in a rectangle $a \leq s \leq b$; $c \leq x \leq d$ such that the left-hand side $g_{\exp }(x)$ of (2) is known accurate to a certain absolute value (error) which defines its deviation from the unknown exact dependence $g(x)$ :

$$
g_{\exp }(x)=g(x) \pm \Delta(x)
$$

The measurement error $\Delta(x)$ allows us to estimate the expected value $\delta>0$ of the so-called left-hand side residual of (2):

$$
\left\|g_{\exp }(x)-g(x)\right\|=\sqrt{\int_{c}^{d} \Delta^{2}(x) d x} \leq \delta .
$$

The knowledge of $\delta$ is required for further controlling the convergence of the approximate solution $f_{\delta}(s)$ to the unknown exact solution $f(s)$.

Finding an approximate solution of (2) consists in finding $f_{\delta}(s) \rightarrow f(s)$ to meet the condition

$$
\left\|K\left[f_{\delta}\right]-g_{\exp }\right\|=\delta .
$$

The above problem of mathematical physics fails to obey the Hadamard criteria because the integral equation with an inaccurately specified left-hand side has an unstable solution with respect to small variations in $g_{\exp }(x)$. It should also be noted that validity of only (5) is insufficient for the arbitrary function $f_{\delta}(s)$ to be a correct approximate solution of (2) with an exact left-hand side (Tikhonov \& Arsenin, 1977). It is the latter fact that makes useless the search for an approximate solution by going over different functions $f_{\delta}(s)$ with subsequent control of residual (5).

To solve the above problem, namely to find a stable approximate solution, an original regularization method for illposed problems was proposed (Tikhonov \& Arsenin, 1977). In the method, the approximate solution to be found is the function $f_{\alpha}(s)$ with a minimum functional

$$
\Phi=\left\|K\left[f_{\alpha}\right]-g_{\exp }\right\|+\alpha \Omega\left[f_{\alpha}\right] .
$$

Here $\alpha$ is a small numerical parameter; $\Omega[f]$ is the stabilizing functional or stabilizer which allows one to find $f_{\alpha}(s)$ in the most general class of functions of certain smoothness.

The form of the stabilizing functional $\Omega$ can be specified from the requirements of continuity and single differentiability of the desired function $f(\epsilon)$. For this purpose, it is sufficient to express this stabilizer as follows:

$$
\Omega[f]=\int_{a}^{b}\left\{f(s)^{2}+\left(\frac{\partial f(s)}{\partial s}\right)^{2}\right\} d s .
$$

Substitution of functional (7) in the Euler equation, which defines that the first variation of functional (6) is equal to zero, gives the integro-differential equation for the desired function $f_{\alpha}(s)$ (Tikhonov \& Arsenin, 1977):

$$
\begin{gathered}
\alpha\left\{f_{\alpha}(s)-\frac{\partial^{2} f_{\alpha}(s)}{\partial s^{2}}\right\}+\int_{a}^{b} f_{\alpha}(t)\left(\int_{c}^{d} K(x, s) K(x, t) d x\right) d t \\
=\int_{c}^{d} K(x, s) g_{\exp }(x) d x
\end{gathered}
$$

the solution of which meets one of the following boundary conditions:

$$
\begin{aligned}
& f(a)=0, f(b)=0 ; \quad f(a)=0, f^{\prime}(b)=0 ; \\
& f^{\prime}(a)=0, f^{\prime}(b)=0 ; \quad f^{\prime}(a)=0, f(b)=0 .
\end{aligned}
$$

These four conditions imposed on the function can always be provided by appropriately choosing a domain of $f(s)$. In our case, we are dealing with electron spectrum (1).

The function $f_{\alpha}(s)$ obtained by solving Eq. (8) depends, in addition to its argument $s$, on the parameter $\alpha$ responsible for accuracy of the approximation $f_{\alpha}(s) \rightarrow f(s)$. The smaller the regularization parameter $\alpha$, the larger the functional $\Omega$ and hence the lower the order of smoothness for $f_{\alpha}(s)$ is admitted in the solution. The parameter $\alpha$ should be chosen so that condition (5) holds true at a given residual $\delta$. Let this value be denoted as $\alpha(\delta)$. Then, the desired approximate solution of ill-posed problem (2) corresponds to $f_{\delta}(s)=f_{\alpha(\delta)}(s)$. The foregoing technique makes it possible to account for the known error in the left-hand side of Eq. (2), thus limiting the final choice of an approximate solution by accuracy of the initial experimental data.

The regularization method described above allows one to find solutions of ill-posed problem (2) without any a priori assumptions on the form of the function $f(\epsilon)$, which are normally involved in spectrum reconstruction (Terebizh, 1995). This property of the method is not at all a guarantee that one can obtain physical solutions of the problem against large systematic or statistical errors in the initial data on the attenuation factor $g_{\text {exp }}(x)$. The generation of nonphysical solutions (e.g., alternating distribution functions) can be indirect evidence for incorrect estimation of initial data error (4).

The second merit of the method is its independence from numerical algorithms which generate the solution $f_{\delta}(s)$. For example, the numerical methods of spectrum reconstruction with so-called basis functions give solutions dependent on the form of these functions and on their number. Sometimes, the result depends also on the way used to construct numerical integration grids (Arzhanikov et al., 2011).

The most valuable property is that the regularization method provides stable approximation to a formally exact solution of Eq. (2) when $\delta \rightarrow 0$. Stability, in this case, means that the varying parameter $\alpha$ causes the function $f_{\alpha}(s)$ to transform gradually without any drastic change in its form. In other words, if we insert an exact function $g(x)$ 
we are bound to automatically come to an exact solution of Eq. (2) at $\alpha \rightarrow 0$.

To disclose other features of the method, we performed test reconstruction of distribution functions on the example of attenuation of a monoenergetic electron beam in aluminum. For the known attenuation function $\tau(\epsilon, x)$ we used semiempirical formulae (Tabata $\&$ Ito, 1975) tested on different materials (atomic numbers $Z$ from 4 to 82 ) and in a wide range of electron energies (from $8 \mathrm{keV}$ to $30 \mathrm{MeV}$ ). The formulae ensured approximation of the experimental attenuation factor to an accuracy of no worse than $3 \%$. For aluminum (atomic number $Z=13$, mass number $A=27$, density $\rho=$ $2.70 \mathrm{~g} / \mathrm{cm}^{3}$ ), the empirical dependence of attenuation of a monoenergetic electron beam with energy $\epsilon(\mathrm{MeV})$ has the form:

$$
\tau(x, \varepsilon) \approx \frac{e^{S(\varepsilon)}+1}{e^{S(\varepsilon)}+\exp \{(S(\varepsilon)+2)(x / R(\varepsilon))\}}
$$

Here, the dimensionless function $S(\epsilon)$ (Tabata \& Ito, 1975) and extrapolated path length $R$ in mm (Weber 1964) for aluminum are calculated by the formulae:

$$
S(\varepsilon) \approx 5.86 \cdot \exp \left\{-\frac{0.72}{1+0.15 \varepsilon^{1.86}}\right\}, \quad R(\varepsilon) \approx \frac{9.37 \varepsilon^{2}}{1+3.83 \varepsilon} .
$$

First, we calculated the discrete dependence of attenuation in aluminum at $E=150 \mathrm{keV}$ by formula (10); the dependence is shown by large rhombuses in Figure 1. These points modeled experimental attenuation of the monoenergetic beam. Then Eq. (8) was solved numerically for different values of the parameter $\alpha$; the smaller the parameter $\alpha$, the smaller the initial data error. Three reconstructed spectra are shown in Figure 2.

As can be seen, some problems arise in reconstructing the monoenergetic beam spectrum; although the spectrum width

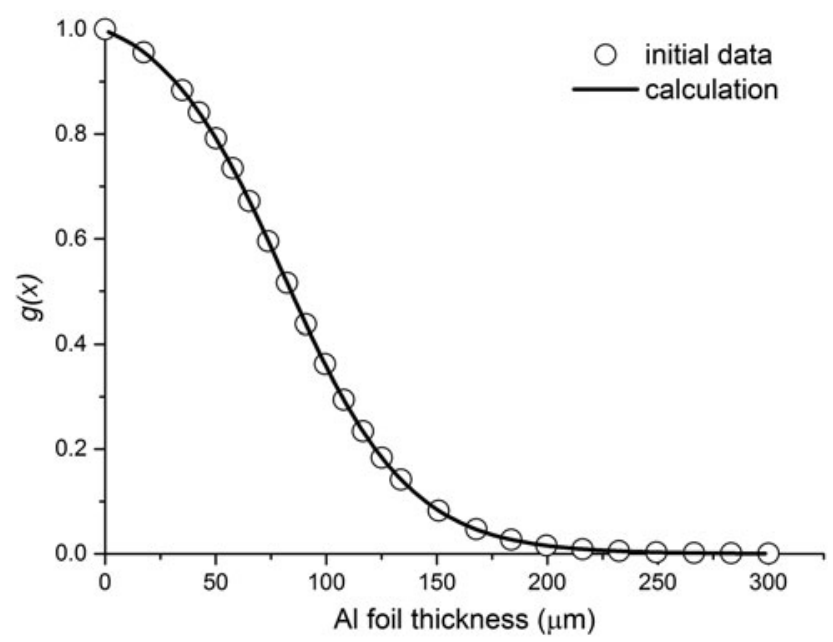

Fig. 1. Beam attenuation for initial data (circles) and calculation at $\alpha=2 \times$ $10^{-9}$ (solid line). decreases with decreasing $\alpha$, alternating wings appear in the spectrum such that the narrower the central part of the spectrum, the more the span of these wings. It is seen in Figure 1 that the beam with a rather broad spectrum attenuates much as the initial monoenergetic beam does. As the parameter $\alpha$ is further decreased (not shown in the figure), the vibration increases in frequency and amplitude, and in the limit, the central peak becomes hardly detectable against this vibration; that is, the desired increase in reconstruction accuracy is limited by the capabilities of the method. In our opinion, this is because the accuracy of numerical solution of Eq. (8) is limited to 12 significant digits and the round-off errors at very small $\alpha\left(<10^{-10}\right)$ begin to exceed the initial data accuracy.

Thus, the regularization method has a significant shortcoming: It is almost incapable of generating stable solutions with sharp peaks, because stabilizing functional (7) strongly "suppresses" such functions. As a result, we obtain not a monoenergetic spectrum but a bell-shaped spectrum, the maximum of which corresponds to the initial beam energy.

The proposed method is efficient for the reconstruction of only rather smooth spectra. However, even for spectra containing narrow lines, the method allows one to determine the characteristic (central) energies of these lines. The test problem also demonstrates that the alternating wings of small amplitude at the interval boundaries are a peculiarity of the method in the reconstruction of non-smooth spectra. Their appearance may just give evidence the presence of narrow lines in a reconstructed spectrum.

\section{EXPERIMENTAL EQUIPMENT AND TECHNIQUES}

The spectra of electron beams released into the atmosphere through a foil window were measured in experiments on two accelerators with vacuum and gas diodes. The accelerator with a vacuum diode was a modified DUET plasmacathode pulsed electron accelerator (Koval et al., 1985); the

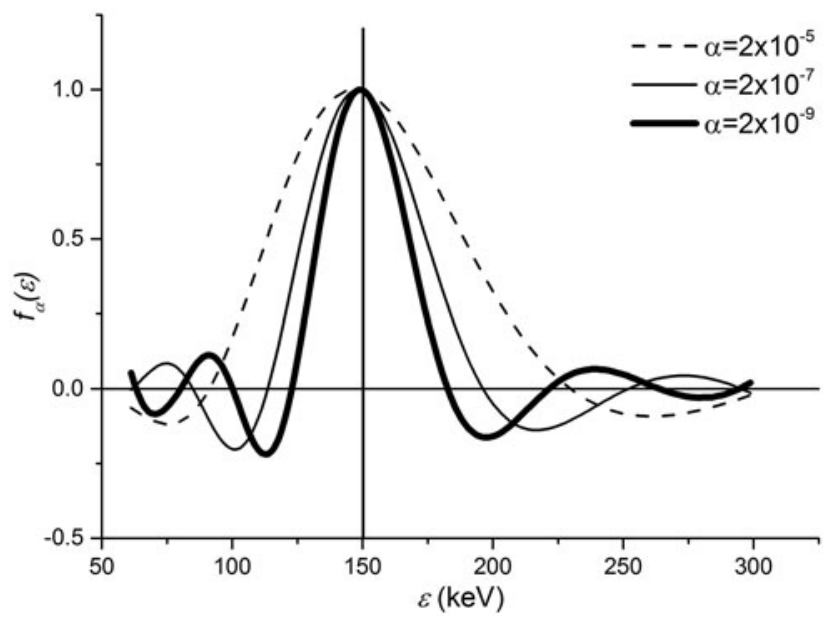

Fig. 2. Spectra reconstructed from the attenuation of a $150 \mathrm{keV}$ (vertical line) monoenergetic $e$-beam for various values of $\alpha$. 
wide-aperture beam released into the atmosphere was of dimensions $750 \times 150 \mathrm{~mm}$. The main characteristics of the accelerator and principle of its operation are described elsewhere (Gielkens et al., 1996; Schanin et al., 2000). Schematic diagram of the accelerator is shown in Figure 3.

The plasma emitter was a hollow semicylinder made of stainless steel. At the edges of the semicylinder, two low-pressure cathode units with cathode spot initiation by a dielectric flashover were fixed. The inner surface of the semicylinder played the role of a common hollow anode for two plasma sources. The constant accelerating voltage applied between the emission grid of the plasma emitter and the output foil window, being the anode of the high-voltage gap, caused extraction of electrons from the plasma and their acceleration to an energy corresponding to the accelerating voltage. The constancy of the accelerating voltage was ensured by properly choosing the capacitance of the highvoltage capacitor bank with respect to the current amplitude and duration in the acceleration gap. Under our experimental conditions, the sag for the capacitor bank was not $>1 \%$. The voltage across the gap was measured with a high-resistance voltage divider and the total current through the diode was measured with a Rogowski coil at the positive terminal of the capacitor bank. The electron beam was injected into the atmosphere via an output window of dimensions $750 \times$ $150 \mathrm{~mm}^{2}$ covered with an aluminum-beryllium (AB-50 composite) foil $40 \mu \mathrm{m}$ thick. The foil was placed on a watercooled support structure of geometric transparency $56 \%$. The main parameters of the accelerator are presented in Table 1.

At a distance of $10 \mathrm{~mm}$ from the output foil, there was a shielded collector of diameter $65 \mathrm{~mm}$ to detect the electron current extracted from the diode gap into the atmosphere. The foil filter thickness was controlled by the varying number of $\mathrm{Al}$ foils, each $9 \mu \mathrm{m}$ thick. Increasing the foil filter thickness decreased the collector current which was recorded until the main (desired) signal compared with the level of electrical noise.

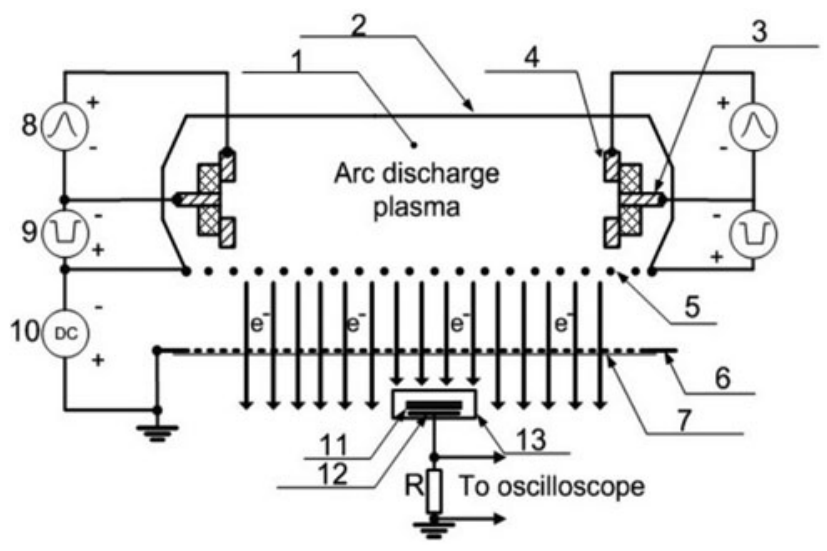

Fig. 3. Electron accelerator with a grid plasma emitter: 1 - plasma emitter; 2, hollow anode; 3 , cathode; 4, trigger electrode; 5 , emission grid; 6 , support grid; 7, output foil window; 8 , discharge power supply; 9 , trigger power supply; 10, high-voltage source; 11 , foil filter; 12 , collector; 13 , shield.
Table 1. The main parameters of the modified DUET plasma-cathode pulsed electron accelerator

\begin{tabular}{ll}
\hline Accelerating voltage $(\mathrm{kV})$ & Up to 200 \\
Emission current $(\mathrm{A})$ & $5-100$ \\
Pulse duration $(\mu \mathrm{s})$ & $5-100$ \\
Pulse repetition frequency $(\mathrm{Hz})$ & up to 50 \\
Beam dimensions $(\mathrm{mm})$ & $750 \times 150$ \\
Cross-sectional beam current inhomogeneity $(\%)$ & \pm 10
\end{tabular}

For generation and diagnostics of an electron beam in a gas diode filled with atmospheric pressure air, a specially designed SLEP-150M accelerator was used (Tarasenko et al., 2011). The pulse rise time was $\sim 250$ ps at a level of $0.1-0.9$ and the full width at half maximum (FWHM) of the voltage pulse with a matched load was $\sim 1 \mathrm{~ns}$. The SLEP-150M accelerator was equipped with an additional transmission line of length $28.8 \mathrm{~cm}$ and four capacitive dividers, making it possible to reproduce the gap voltage from incident and reflected voltage waves (Baksht et al., 2010; Tarasenko, 2011; Tarasenko et al., 2011). The discharge current was measured with a current shunt composed of chip resistors. The output section of the SLEP-150M accelerator, including the additional transmission line, gas diode with a thin-walled tubular cathode of diameter $6 \mathrm{~mm}$ (Tarasenko et al., 2004), and collector with a time resolution of $\sim 80 \mathrm{ps}$, is shown in Figure 4.

The plane anode of the gas diode was an aluminum foil $10 \mu \mathrm{m}$ thick reinforced with a diaphragm or a grid. The gap between the cathode and anode was $12 \mathrm{~mm}$. The gas diode was filled with atmospheric pressure air. The wave resistance of the transmission line was $100 \Omega$. The SLEP-150M accelerator produced voltage pulses with an incident wave amplitude of $130 \mathrm{kV}$ in the transmission line. The accelerator could also be operated with a vacuum diode for which the cathode remained the same and the diode was pumped by a forevacuum pump. The interelectrode gap for the SLEP-150M accelerator operated with a vacuum diode was decreased to $4 \mathrm{~mm}$.

For measuring the beam attenuation, aluminum foil filters of thickness $10,50,100$, and $200 \mu \mathrm{m}$ or sets of aluminum foils with the least number in a filter were used.

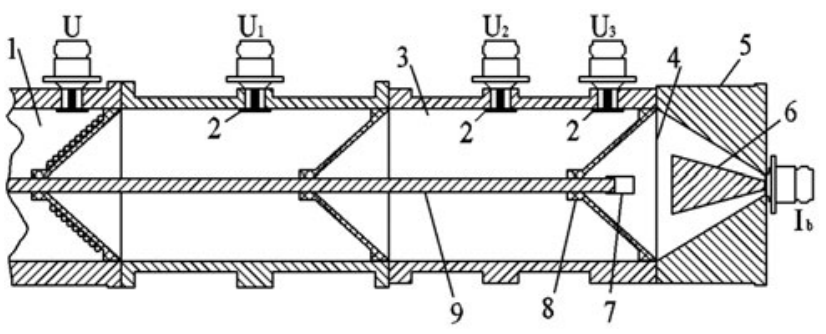

Fig. 4. Output section of the SLAP-150M accelerator and collector: 1, short transmission line; 2 , capacitive voltage dividers; 3 , additional transmission line; 4 , foil with a diaphragm; 5 , collector case; 6 , collector receiving part; 7 , cathode; 8 , insulator of the gas diode; 9 , central conductor of the coaxial line. 
a)

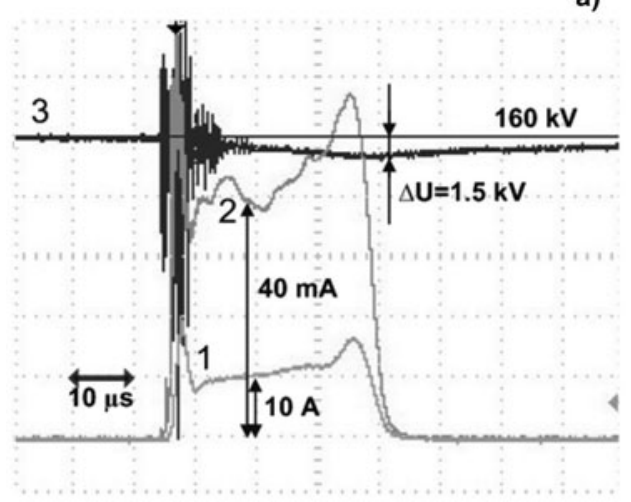

b)

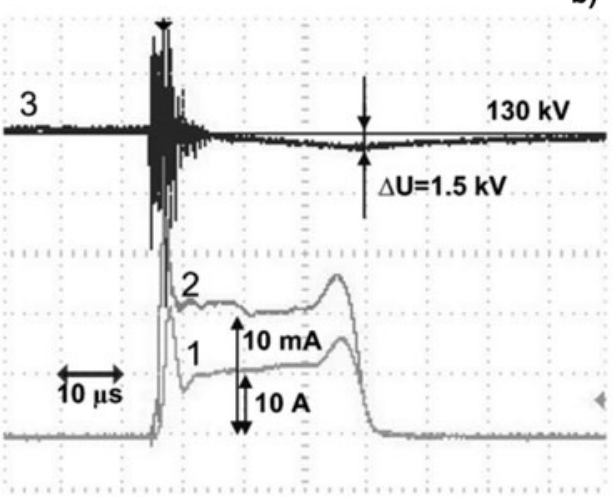

Fig. 5. Waveforms of the electron beam current in the acceleration gap (1), current at the collector after beam passage through a foil filter of thickness $45 \mu \mathrm{m}$ (2), and voltage sag at the high-voltage capacitor bank (3) at a vacuum diode voltage of $160 \mathrm{kV}$ (a) and $130 \mathrm{kV}$ (b).

Signals of the voltage, SAEB, and discharge current were transmitted to a Tektronix TDS6604 digital oscilloscope $(6 \mathrm{GHz}, 20 \mathrm{GS} / \mathrm{s})$. The beam current pulses were recorded using a high-frequency cable of length $1 \mathrm{~m}$ (Radiolab RG58-A/U), N-type connectors (Suhner 11 N-50-3-28/ $133 \mathrm{NE}$ ), and SMA connectors (Radiall R125.075.000).

\section{RESEARCH RESULTS}

\subsection{Spectra Reconstructed from Beam Attenuation on the DUET Accelerator with a Vacuum Diode}

Figure 5 shows typical waveforms of the total current in the acceleration gap (1), current at the collector after electron beam passage through a foil filter of thickness $45 \mu \mathrm{m}$ (2), and voltage sag at the high-voltage capacitor bank (3) for different voltages across the acceleration gap.

As noted above, the voltage applied to the acceleration gap was constant. The beam parameters were controlled by the varying parameters of plasma generation in the plasma emitter. So, the current amplitude, duration, and pulse repetition frequency in the acceleration gap were controlled by the varying discharge current amplitude, duration, and pulse repetition frequency (irrespective of the accelerating voltage). In the experiments, the FWHM of the beam current pulse was $\sim 35 \mu$ s. Because the signal of the sag across the capacitor bank was interfered within the first $10 \mu \mathrm{s}$, the collector currents used to plot the attenuation curve were also taken within $10 \mu$ s after the beginning of the pulse. To decrease the influence of the voltage sag across the high-voltage capacitor bank on the energy homogeneity of the electron beam and to simplify the calculations of its energy spectrum, the current in the acceleration gap was relatively low and was $10 \mathrm{~A}$.

Figure 6 shows experimental points of the electron beam attenuation at different initial accelerating voltages across the vacuum diode.

The highest current amplitudes at the collector were recorded at the maximum initial accelerating voltage. It is apparent that for the reliable detection of the collector current downstream of the foil filter, it is required to choose the maximum filter thickness depending on the accelerating voltage.

Figures 7 and 8 show experimental (open circles) and calculated (solid lines) attenuation curves and spectra of fast electrons reproduced by the regularization method for an initial vacuum diode voltage of 130 and $160 \mathrm{kV}$.

The attenuation curves of the beam current at a vacuum diode voltage of 130 and $160 \mathrm{kV}$ were also calculated using the reconstructed electron spectra. The calculated attenuation curves are shown by solid lines in Figures $7 \mathrm{a}$ and $8 \mathrm{a}$. At a voltage across the vacuum gap of 130 and $160 \mathrm{kV}$, electrons with energies $<40 \mathrm{keV}$ are not detected in the spectrum reconstructed. The high-energy tail present in the electron energy distribution at a beam current pulse duration of tens of microseconds can be explained by the above-mentioned shortcomings of the regularization, which smoothes the electron spectrum. The maximum in the electron energy distribution is lower than the electron energy defined as $e U_{0}$, where $U_{0}$ is the initial accelerating voltage across the vacuum diode. This is because the average beam energy decreases in the

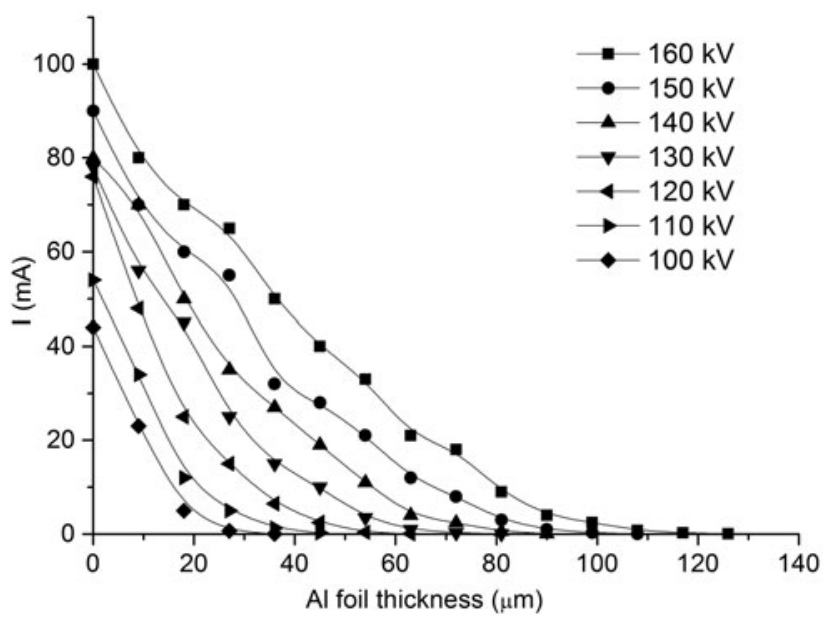

Fig. 6. Experimental points of the electron beam attenuation on the DUET accelerator at different initial accelerating voltages. 

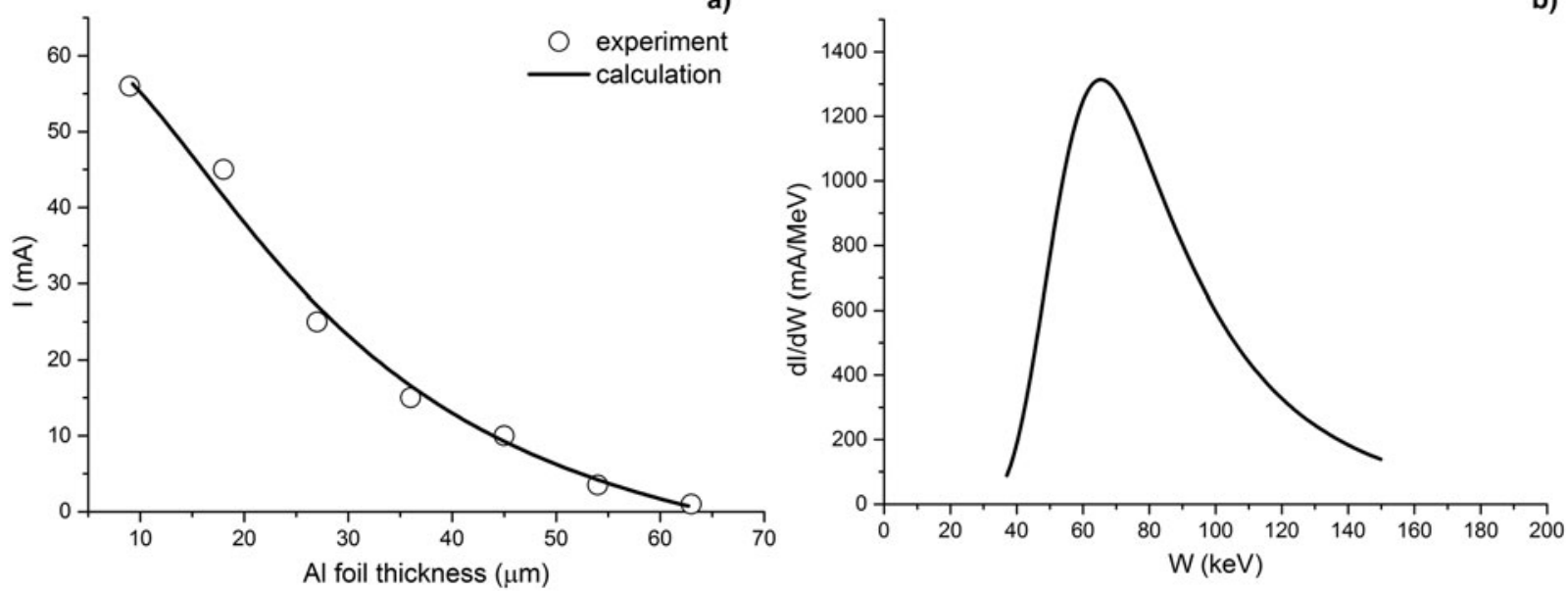

Fig. 7. Attenuation curve of the electron beam current $I$ (a) and electron spectrum at the output of the DUET accelerator (b) for a vacuum diode voltage of $130 \mathrm{kV}$.
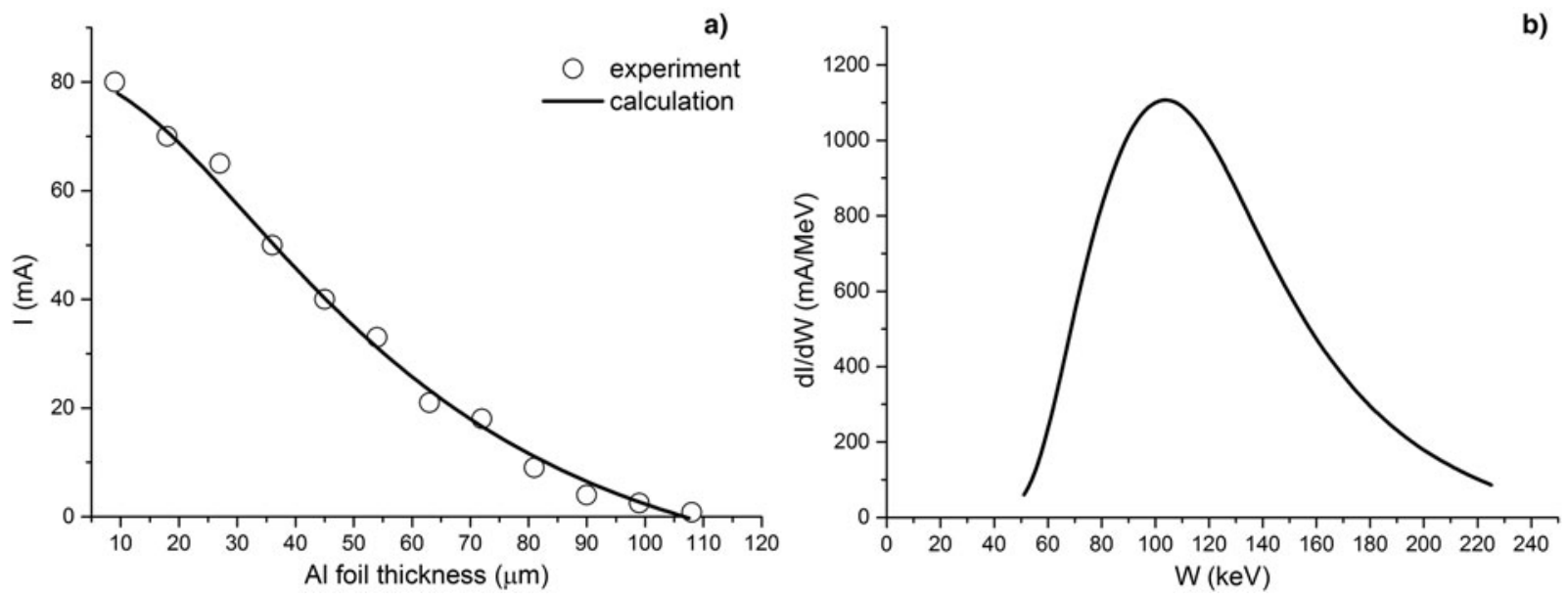

Fig. 8. Attenuation curve of the electron beam current $I$ (a) and electron spectrum at the output of the DUET accelerator (b) for a vacuum diode voltage of $160 \mathrm{kV}$.

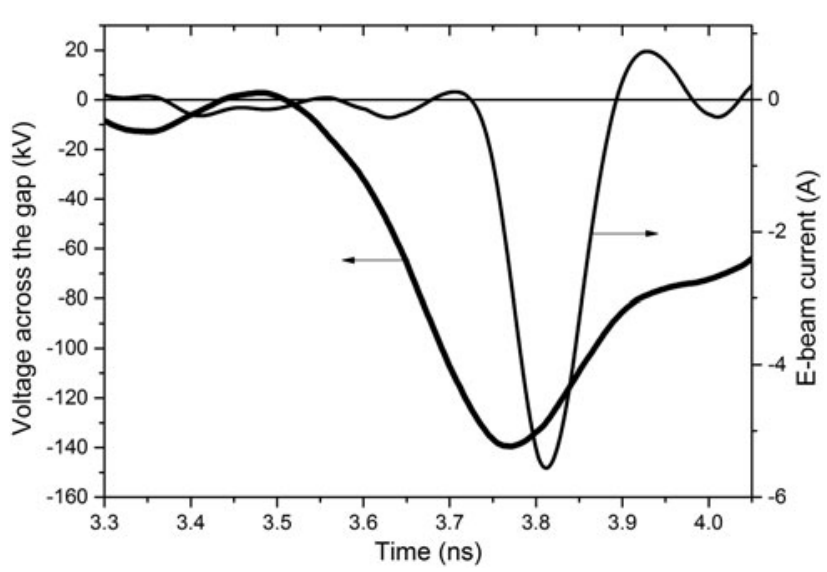

Fig. 9. Waveforms of the voltage and electron beam current at the output of the SLEP-150M accelerator with a tubular cathode and $12 \mathrm{~mm}$ interelectrode gap. output foil of thickness equivalent to $20 \mu \mathrm{m}$ of pure $\mathrm{Al}$ foil and also because the collector is placed in atmospheric air at a distance of $10 \mathrm{~mm}$ from the anode foil. Calorimetric analysis shows that as the beam with an initial energy of $160 \mathrm{keV}$ travels a distance of $1 \mathrm{~cm}$ in atmospheric air, its average energy decreases by $5-7 \mathrm{keV}$. It should also be noted that when the set of foils is replaced by a single foil of the same thickness, the collector current increases by 5\%. The data for beam energy loss agree with those obtained on an electron accelerator with a hot cathode and beam release into the atmosphere (Grigor'ev \& Shanturin, 1979; Grigor'ev \& Stepanov, 1982).

\subsection{Spectra Reconstructed from Beam Attenuation on the SLEP-150M Accelerator with a Gas Diode}

The spectra of runaway electrons produced in gas diodes at increased pressures are much more difficult to measure due 

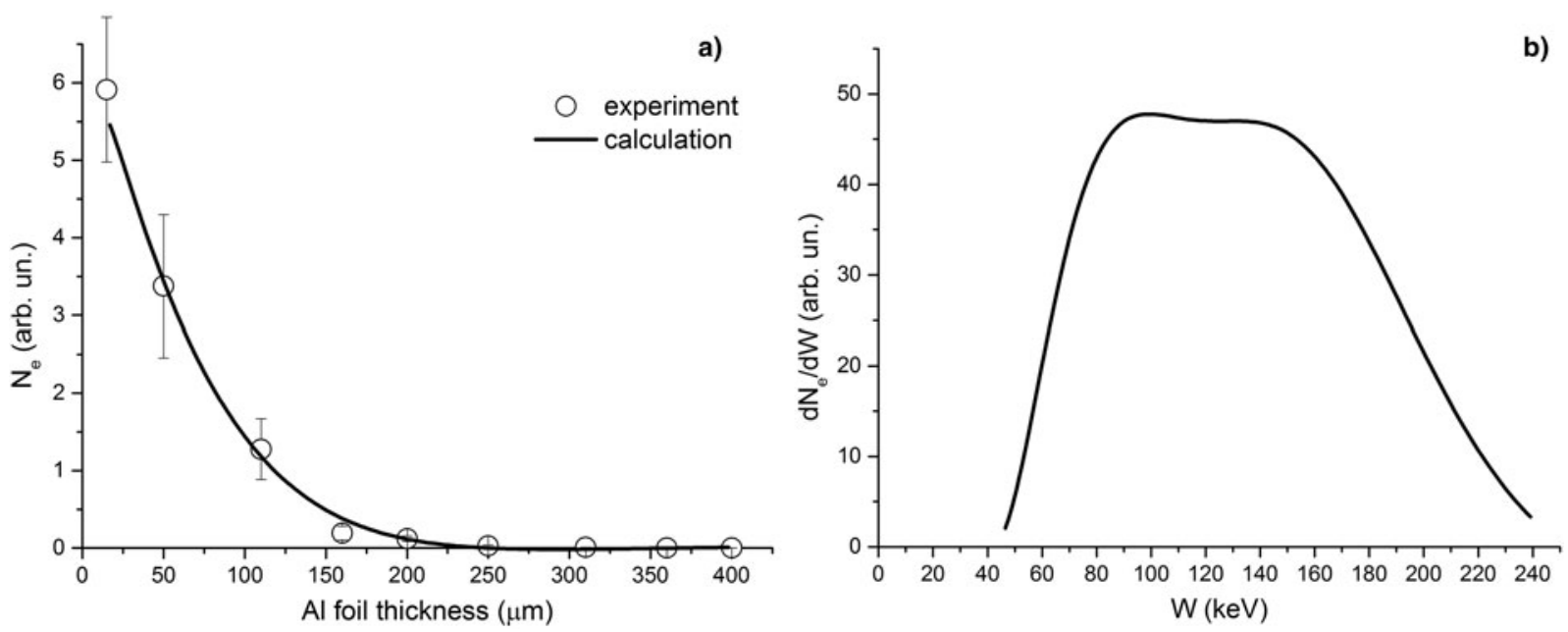

Fig. 10. Beam attenuation curve (a) and electron spectrum at the output of the SLEP-150M accelerator (b) for a gas diode filled with atmospheric pressure air and $12 \mathrm{~mm}$ interelectrode gap.

to short SAEB durations (tens and hundreds of picoseconds). Therefore, the measurement results depend largely on the quality of diagnostic equipment.

Research data on runaway electrons produced in the gas diode of the SLEP-150M accelerator are presented in Figures 9 and 10. Figure 9 shows waveforms of the gap voltage and beam current.

The width of the beam current pulse downstream of the entire anode foil in atmospheric pressure air was $\sim 100$ ps. The maximum voltage across the gap with no breakdown reached $\sim 270 \mathrm{kV}$. The SAEB was detected by the collector at the maximum gap voltage.

Figure 10 shows experimental (open circles) and calculated (solid lines) attenuation curves and spectrum of fast electrons in atmospheric pressure air reconstructed by the regularization method for a tubular cathode.

As can be seen, the spectrum contains two local maxima at 98 and $130 \mathrm{keV}$. In these experiments, the maximum voltage

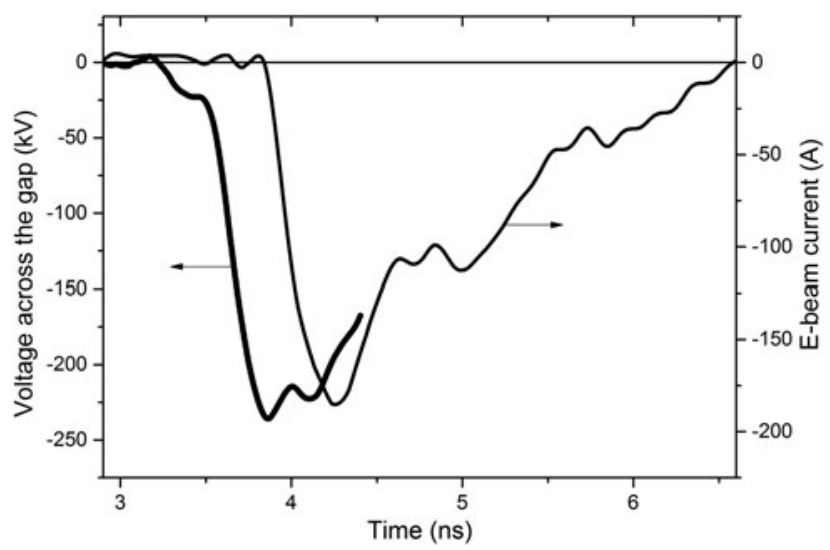

Fig. 11. Waveforms of the voltage and beam current at the output of the SLEP-150M accelerator for a vacuum diode with a tubular cathode and $4 \mathrm{~mm}$ interelectrode gap. across the gas diode with a tubular cathode was $140 \mathrm{kV}$. The presented results agree with the data reported elsewhere (Baksht et al., 2008a; 2009; 2010; Tarasenko, 2011; Tarasenko et al., 2005; 2008; 2011; 2013). In the papers cited, the SAEB spectrum in atmospheric pressure air also revealed several groups of electrons. Electrons of the first group had energies $T_{1}<e U_{\mathrm{m}} / 3$; for detection of low-energy electrons, very thin anode foils and metal grids with a mesh size of $<0.2 \times 0.2 \mathrm{~mm}$ were used. Electrons of the second (main) group had energies $e U_{\mathrm{m}} / 3<T_{2}<e U_{\mathrm{m}}$. This result was confirmed by additional experiments on SAEB deflection in a magnetic field (Baksht et al., 2008b) and by measurements with a time-of-flight spectrometer (Baksht et al., 2010; Tarasenko et al., 2013). Electrons of the third group had energies $T_{3}>e U_{\mathrm{m}}$. The number of electrons in each group depended on experimental conditions, voltage pulse parameters, and cathode and diode designs. Increasing the number of electrons in the third group required a decrease in voltage pulse rise time and an increase in cathode curvature radius (Baksht et al., 2010). However, under near-optimal SAEB generation conditions the number of electrons of the third group was rather small and was not $>10 \%$ of the total number of fast electrons downstream of the anode foil.

\subsection{Spectra Reconstructed from Beam Attenuation on the SLEP-150M Accelerator with a Vacuum Diode}

Experiments on the SLEP-150M accelerator were also performed with a vacuum diode. For increasing the beam current, the interelectrode gap was decreased to $4 \mathrm{~mm}$. Waveforms of the voltage across the gap and beam current are presented in Figure 11.

It is seen that for the vacuum diode, the FWHM of the current pulse increased to $\sim 1 \mathrm{~ns}$. The voltage across the gap reached its maximum and the voltage pulse decayed more gradually compared with the gas diode experiments. 

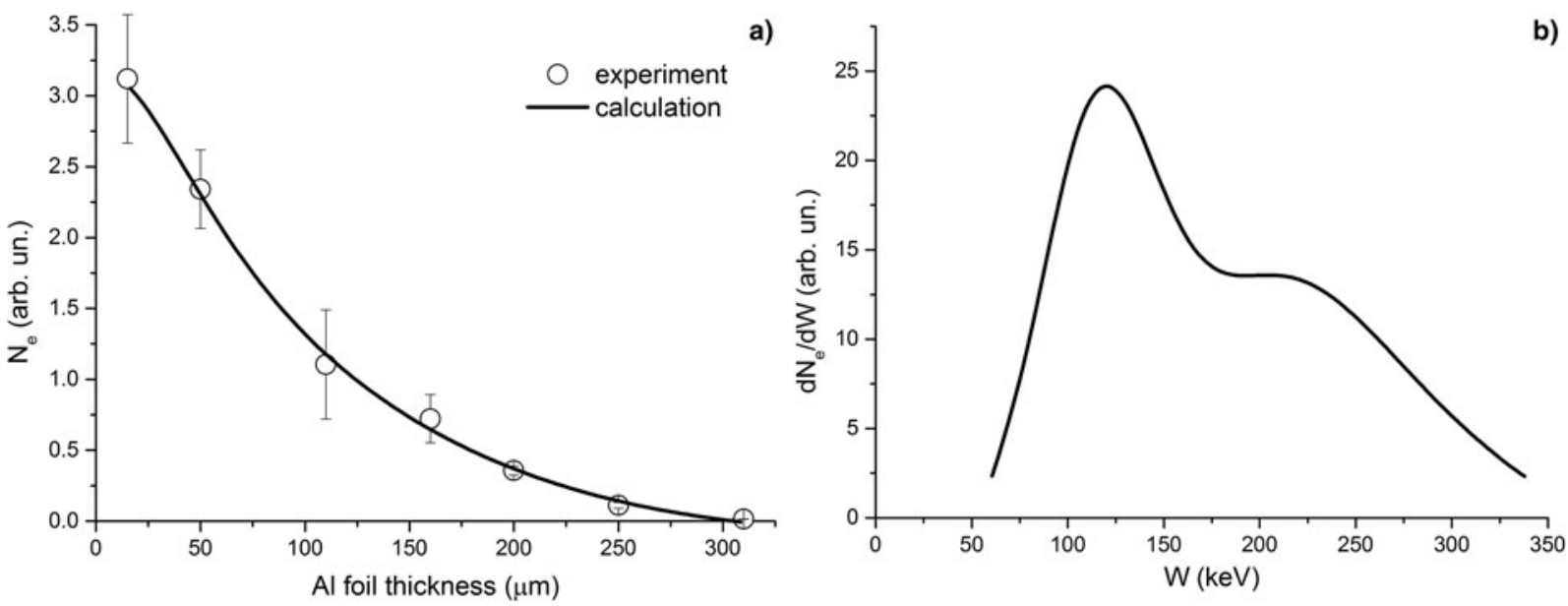

Fig. 12. Beam attenuation curves (a) and electron spectrum at the output of the SLEP-150M accelerator (b) for a vacuum diode and $4 \mathrm{~mm}$ interelectrode gap.

Figure 12 shows experimental (open circles) and calculated (solid lines) attenuation curves and spectrum of fast electrons at the output of the SLEP-150M accelerator with a vacuum diode.

The reconstructed spectrum reveals a single maximum at $\sim 120 \mathrm{keV}$ and a group of electrons with an energy of $\sim 200 \mathrm{keV}$, which roughly corresponds to the voltage pulses and diode current (Fig. 11).

\section{DISCUSSION OF THE RESULTS}

The results reported in the paper demonstrate the possibility to determine the electron energy from beam attenuation curves for vacuum and gas diodes. With voltage pulses of tens of microseconds applied to the vacuum diode the electron beam spectrum has a single maximum, which is expectable. With nanosecond voltage pulses the electron spectrum extends greatly toward lower energies than the energy corresponding to the maximum diode voltage. This is due to the generation of fast electrons during the voltage rise and decay. Note that electrons with anomalous energy in the vacuum diodes escaped detection; the high-energy tail in the spectrum in Figure $12 \mathrm{~b}$ can be attributed to the peculiarities of the method used.

The main distinction in the spectra of fast electrons produced in gas-filled diodes is the presence of two or three groups of SAEB electrons. Electrons with increased energy in the gas diode of the SLEP-150M accelerator were also detected in measurements with a time-of-flight spectrometer (Baksht et al., 2009). It should be noted that the number of electrons with an energy $T_{3}>e U_{\mathrm{m}}$, unlike those of the other two groups, varied greatly from pulse to pulse. This can be explained by unstable electron self-acceleration conditions at the ionization wave front and hence by their variation from pulse to pulse (Askaryan, 1973). The conducted study suggests that there is probably an essential error in the measurements of runaway electron spectra in atmospheric pressure air by Babich and colleagues (Babich \& Loiko, 1985; 1991; Babich et al., 2014).

\section{CONCLUSION}

The conducted study confirms that the Tikhonov regularization of ill-posed problems on minimum a priory assumptions allows one to reconstruct the spectra and energy characteristics of an electron beam from its attenuation in foils of different thickness.

\section{ACKNOWLEDGMENT}

The work was performed under RSF Project No. 14-29-00052.

\section{REFERENCES}

Afanasiev, V.N., Bychkov, V.B., Lartsev, V.D., Pudov, V.P., Solomonov, I., Shunailov, S.A., Generalova, V. \& Gromov, A.A. (2005). Parameters of the electron beams generated by the RADAN-220 and RADAN-EXPERT accelerators. Instrum. Exp. Tech. 48, 641-645.

Arzhanikov, A.V., Makarov, M.A., Sinitsky, S.L. \& Stepanov, V.D. (2011). Energy spectrum of electrons in flow from plasma column heated by REB at GOL-3 facility. Fusion Sci. Technol. 59, 304-306.

Askaryan, G.A. (1973). About new possibilities on particle acceleration up to high energies. Trudy FIAN 66, 66-72.

BABICH, L.P. \& LoIKo, T.V. (1985). Energy spectra and time parameters of the runaway electrons at a nanosecond breakdown in dense gases. Tech. Phys. 55, 956-958.

BABICH, L.P. \& LoIKo, T.V. (1991). Runaway electrons at high voltage nanosecond discharges in sulphur hexafluoride at pressure of 1 atm. Tech. Phys. 61, 153-155.

BABICH, L.P., LoIKo, T.V. \& Rodigin, A.V. (2014). Calibration of detectors of ionizing emissions by means of a subnanosecond runaway electron beam generated by discharge in open atmosphere at high overvoltages. Instrum. Exp. Tech. 57, 248-254. 
Baksht, E.Kh., Burachenko, A.G., Erofeev, M.V., Kostyrya, I.D., LomaeV, M.I., RybKa, D.V. \& TARASENKo, V.F. (2008b). Effect of a transverse magnetic field on the generation of electron beams in the gas-filled diode. Tech. Phys. 53, 804-807.

Baksht, E.Kh., Burachenko, A.G., Kozhevnikov, V.Y., Kozyrev, A.V., Kostyrya, I.D. \& Tarasenko, V.F. (2010). Spectrum of fast electrons in a subnanosecond breakdown of air-filled diodes at atmospheric pressure. J. Phys. D: Appl. Phys. 43, 305201.

Baksht, E.Kh., Burachenko, A.G., Kozyrev, A.V., Kostyrya, I.D., Lomaev, M.I., Petin, A.V., Rybka, D.V., Tarasenko, V.F. \& Shlyakhtun, S.V. (2009). Spectra of electrons and X-ray photons in a diffusive nanosecond discharge in air under atmospheric pressure. Tech. Phys. 54, 47-55.

Baksht, E.Kh., Burachenko, A.G., Lomaev, M.I., Rybka, D.V. \& TARASENKO, V.F. (2008a). Generation of runaway electron subnanosecond pulses in nitrogen and helium at a voltage of $25 \mathrm{kV}$ across the gap. Tech. Phys. 53, 93-98.

Baksht, E.H., Kostyrya, I.D., Lipatov, E.I., Lomaev, M.I., RybKa, D.V. \& TARASENKo, V.F. (2007). Excess-energy electrons in a nanosecond electron beam from a vacuum diode. Tech. Phys. 52, 489-494.

Bugaev, S.P., Koshelev, V.I. \& Timofeev, M.N. (1974). Electron-energy spectrum in high-current vacuum diode. $Z h$. Tekh. Fiz. 44, 1917-1921.

Gielkens, S.W.A., Peters, P.J.M., Witteman, W.J., Borovikov, P.V., Stepanov, A.V., Tskhai, V.N., Zavjalov, M.A., GusheNETS, V.I. \& Koval, N.N. (1996). A long-pulse $300 \mathrm{keV}$ electron gun with a plasma cathode for high-pressure gas lasers. Rev. Sci. Instrum. 67, 2449-2452.

Gloeckler, G. \& Hsieh, K.C. (1979). Time-of-flight technique for particle identification at energies from 2 to $400 \mathrm{keV} /$ nucleon. Nucl. Instrum. Methods 165, 537-544.

Grigor'ev, Yu.V. \& Shanturin, L.P. (1979). Four-electrode electron gun with an output flow of cross-section $10 \times 80 \mathrm{~cm}^{2}$ into the atmosphere. Prib. Tekh. Eksp. 4, 194-196. In Russian.

Grigor'ev, Yu.V. \& Stepanov, A.V. (1982). Measurement of the electron current in air at an electron energy of $60-130 \mathrm{keV}$. Prib. Tekh. Eksp. 5, 124-126. In Russian.

Hegeler, F., Friedman, M., Myers, M.C., Sethian, J.D. \& SwaneKAMP, S.B. (2002). Reduction of edge emission in electron beam diodes. Phys. Plasmas 9, 4309-4315.

Khudyakova, L.N., Gutnikova, E.K. \& Tarasova, L.V. (1964). Hard X-radiation component of pulsed X-ray tube. Zh. Tekh. Fiz. 34, 2044-2047.

Koval, N.N., Kreindel, Yu.E., Tolkachev, V.S. \& Schanin, P.M. (1985). The effect of gas on the development of a vacuum arc with a hollow anode. IEEE Trans. Electr. Insul. El-20, 735-737.

Li, Y.T., Yuan, X.H., Xu, M.H., Zheng, Z.Y., Sheng, Z.M., Chen, M., Ma, Y.Y., Liang, W.X., Yu, Q.Z., Zhang, Y., Liu, F., WANG, Z.H., WeI, Z.Y., ZhaO, W., Jin, Z. \& ZhANG, J. (2006). Observation of a fast electron beam emitted along the surface of a target irradiated by intense femtosecond laser pulses. Phys. Rev. Lett. 96, 165003.

Mangles, S.P.D., Murphy, C.D., Najmudin, Z., Thomas, A.G.R., Collier, J.L., Dangor, A.E., Divall, E.J., Foster, P.S., GalLACher, J.G., HoOKer, C.J., Jaroszynski, D.A., LANGley, A.J., Mori, W.B., Norreys, P.A., Tsung, F.S., Viskup, R., Walton,
B.R. \& Krushelnick, K. (2004). Monoenergetic beams of relativistic electrons from intense laser-plasma interactions. Nature 431, 535-538.

Mesyats, G.A., Osipov, V.V. \& Tarasenko, V.F. (1995). Pulsed Gas Lasers. Bellingham: SPIE Optical Engineering Press.

Mesyats, G.A. (2005). Pulsed Power. New York: Springer Science and Business Media, Inc.

Mesyats, G.A., Reutova, A.G., Sharypov, K.A., Shpak, V.G., ShUNAILOV, S.A. \& YALANDIN, M.I. (2011). On the observed energy of runaway electron beams in air. Laser Part. Beams 29, 425-435.

Schanin, P.M., Koval, N.N., Tolkachev, V.S. \& Gushenets, V.I. (2000). Plasma-emitter electron accelerators. Rus. Phys. J. 43, 427-431.

Shao, T., Tarasenko, V.F., Zhang, C., Baksht, E.Kh., Yan, P. \& ShuT'Ko, Yu.V. (2012). Repetitive nanosecond-pulse discharge in a highly nonuniform electric field in atmospheric air: X-ray emission and runaway electron generation. Laser Part. Beams 30, 369-378.

TAвAтA, T. \& ITo, R. (1975). A generalized empirical equation for the transmission coefficient of electrons. Nucl. Instrum. Methods 127, 429-434.

TARASENKo, V.F. (2011). Parameters of a supershort avalanche electron beam generated in atmospheric-pressure air. Plasma Phys. Rep. 37, 409-421.

Tarasenko, V.F., BaKsht, E.Kh., Burachenko, A.G., Kostyrya, I.D., Lomaev, M.I. \& RyBKA, D.V. (2008). Supershort avalanche electron beam generation in gases. Laser. Part. Beams 26, 605-617.

Tarasenko, V.F., Baksht, E.Kh., Burachenko, A.G., Kostyrya, I.D. \& RYBKA, D.V. (2013). Energy of electrons generated during a subnanosecond breakdown in atmospheric-pressure air. Plasma Phys. Rep. 39, 592-599.

Tarasenko, V.F., Kostyrya, I.D., Baksht, E.Kh. \& Rybka, D.V. (2011). SLEP-150M compact supershort avalanche electron beam accelerator. IEEE Trans. Dielectr. Electr. Insul. 18, 1250-1255.

Tarasenko, V.F., ShPak, V.G., Shunailov, S.A. \& Kostyrya, I.D. (2005). Supershort electron beam from air filled diode at atmospheric pressure. Laser Part. Beams 23, 545-551.

Tarasenko, V.F., Skakun, V.S., Kostyrya, I.D., Alekseev, S.B. \& OrLOVSKII, V.M. (2004). On formation of subnanosecond electron beams in air under atmospheric pressure. Laser Part. Beams 22, 75-82.

TEREBIZH, V.Y. (1995). Image restoration with minimum a priori information. Phys. - Usp. 38, 137-167.

Tikhonov, A.N. \& Arsenin, V.Y. (1977). Solutions of Ill-Posed Problems. Washington: Winston \& Sons.

Verbeeck, J., Tian, H. \& Schattschneider, P. (2010). Production and application of electron vortex beams. Nature 467 301-304.

Weber, K.-H. (1964). Eine einfache Reichweifer-energieBeziehung für electronen im energiebereich von $3 \mathrm{keV}$ bis $3 \mathrm{MeV}$. Nucl. Instrum. Methods 25, 261-264.

Yatom, S., Verselman, V., Gleizer, J.Z. \& Krasik, Ya.E. (2011). Space- and time-resolved characterization of nanosecond time scale discharge at pressurized gas. J. Appl. Phys. 109, 073312.

Zhang, C., Tarasenko, V.F., Shao, T., Beloplotov, D.V., Lomaev, M.I., SOROKIN, D.A. \& YAN, P. (2014). Generation of super-short avalanche electron beams in $\mathrm{SF}_{6}$. Laser Part. Beams 32 , 331-341. 